\title{
Spotlight on pembrolizumab in the treatment of advanced melanoma
}

This article was published in the following Dove Press journal:

Drug Design, Development and Therapy

4 June 2015

Number of times this article has been viewed

\section{Thanashan Rajakulendran' \\ David N Adam²}

'Department of Medicine, Division of Dermatology, Postgraduate Medical Education, ${ }^{2}$ Department of Medicine, Division of Dermatology, St Michael's Hospital, University of Toronto, Toronto, ON, Canada
Correspondence: Thanashan

Rajakulendran

Division of Dermatology, Sunnybrook Health Sciences Centre, 2075 Bayview Avenue, Suite MI-700, Toronto, Ontario, M4N 3M5, Canada

Email t.rajakulendran@mail.utoronto.ca

David N Adam

Department of Medicine, Division of Dermatology, St Michael's Hospital, 30 Bond Street, Fourth Floor Cardinal Carter North, Toronto, ON M5B IW8, Canada

Email david.adam@utoronto.ca

\begin{abstract}
Metastatic melanoma is an aggressive cancer with a poor prognosis. Many approved therapies often do not achieve durable responses in patients. This underscores the need for novel therapeutic strategies. Recruiting a robust immune response is an important antineoplastic treatment strategy. Immune checkpoints offer a molecular target for modulating the immune response and a promising therapeutic target in metastatic melanoma. Here we discuss the recent approval of pembrolizumab by the US Food and Drug Administration for the treatment of metastatic melanoma and its impact on future management of the disease.
\end{abstract}

Keywords: pembrolizumab, melanoma, immune checkpoint, PD-1, PD-L1

\section{Introduction}

Metastatic melanoma is an aggressive and difficult to treat cancer. While surgery and radiation therapy play a vital role in the palliation of symptoms, systemic therapy remains the cornerstone of treatment. ${ }^{1}$ Until recently, systemic therapy largely consisted of dacarbazine, an alkylating agent, which was first approved by the US Food and Drug Administration (FDA) in 1975. The 10-year survival rate for patients with metastatic melanoma is $<10 \%$ with this treatment approach, ${ }^{2}$ underscoring the need for new therapies with improved efficacy.

Great strides have been made in elucidating the key steps in melanomagenesis in the last decade. This has expanded the armamentarium with a host of medications that are rationally designed and target key molecular pathways. Advances in targeted therapeutics aimed at specific defects in tumor initiation and progression have contributed profoundly toward achieving durable responses in some patients. Notably, targeting the mitogen-activated protein (MAP) kinase signaling pathway with small-molecule inhibitors has been encouraging since the outset. Response rates vary from $\sim 60 \%$ to $70 \%$ for patients treated with inhibitors of the MAP kinase cascade, and these agents provide a survival advantage over traditional chemotherapy. ${ }^{3,4}$ However, in many patients, these agents often become ineffective over a period of several months as molecular resistance develops. ${ }^{5}$ Furthermore, only $\sim 50 \%-70 \%$ of patients with specific mutations in the $B R A F$ gene of the MAP kinase cascade can benefit from these inhibitors with a median duration of response of 5-7 months on BRAF inhibitor monotherapy. ${ }^{6}$ Combination therapy with multiple inhibitors targeting BRAF and MEK in the MAP kinase cascade improves response rates and progression-free survival, but this tactic is also prone to the development of resistance with prolonged treatment. ${ }^{7}$ As such, tremendous efforts are ongoing toward developing novel approaches for the effective treatment of advanced melanomas.

The immune system is well recognized for its role in cancer surveillance. ${ }^{8}$ Enlisting a robust immune response is an important antineoplastic treatment strategy. Immune

submit your manuscript | www.dovepress.com 
checkpoints offer a molecular target for modulating the immune response in cancers. ${ }^{9}$ In this regard, the cytotoxic T-lymphocyte-associated antigen (CTLA)-4 and programmed cell death (PD)-1 receptor and its ligands (PD-L1 and PD-L2) have gained consideration as targets in antineoplastic drug design ${ }^{10}$ (Figure 1). Both pathways suppress the activity of T-lymphocytes ( $\mathrm{T}$ cells), which normally play a vital role in tumor immune surveillance. An active area of research is the development of immune checkpoint inhibitors that block CTLA-4 and PD-1 with the hope that these strategies can lead to durable responses in patients with a wide range of cancers, including melanoma. ${ }^{9}$ PD-1 and PD-L1 inhibitors are currently in many stages of clinical investigation, and the anti-PD-1 antibody, pembrolizumab, is the latest addition to the treatment options in melanoma. ${ }^{11}$

\section{Development of immune checkpoint inhibitors}

$\mathrm{T}$ cells have the potential to recognize cancer-related antigens as nonself and eliminate these transformed cells. Thus, mechanisms that allow cancer cells to bypass this immune surveillance enable unchecked tumor growth. One such mechanism by which cancer cells limit the host immune response is via upregulation of PD-L1 and its ligation to PD-1 on antigen-specific CD8 ${ }^{+} \mathrm{T}$ cells. ${ }^{10}$ This is termed adaptive immune resistance ${ }^{12}$ (Figure 1).

The interaction of PD-L1 expressed on a cancer cell with the PD-1 receptor on $\mathrm{T}$ cells leads to immune suppression and escape from tumor immune surveillance. Therapeutic interventions that prevent the PD-1 to PD-L1 interaction would be expected to restore an active immune response against tumors. An analogous pathway on $\mathrm{T}$ cells is the CTLA-4 interaction with B7 that similarly leads to immune suppression and has previously been targeted by antineoplastic agents ${ }^{13}$ (Figure 1). The CTLA-4 and PD-1 pathways differ in their molecular details that lead to suppression of activation of $\mathrm{T}$ cells.

CTLA-4 was the first immune checkpoint receptor to have a targeted therapeutic. Ipilimumab was approved by the FDA in 2011 for the treatment of metastatic melanoma. ${ }^{14}$ In contrast to CTLA-4, which regulates T cells at the level of priming activation upon antigen presentation, PD-1 regulates immunity at several downstream stages of the immune response, including its effect on effector T-cell activity in peripheral tissues, which is central to immune surveillance. Therefore, targeting PD-1 could offer a more robust response than targeting CTLA-4.

\section{FDA approval of pembrolizumab}

Pembrolizumab was given fast-tracked approval by the FDA in September 2014 for the treatment of patients with metastatic melanoma who failed ipilimumab treatment and, if $B R A F$ mutation was positive, also failed treatment with a BRAF inhibitor. While a number of PD-1/PD-L1 inhibitors are under development (Table 1), pembrolizumab is the first PD-1 inhibitor to be approved by the FDA in melanoma. It is a monoclonal antibody that binds to PD-1 and prevents its interaction with PD-L1. ${ }^{11}$ This allows T-cell activation to take place, without interference from tumor-elaborated PD-L1.

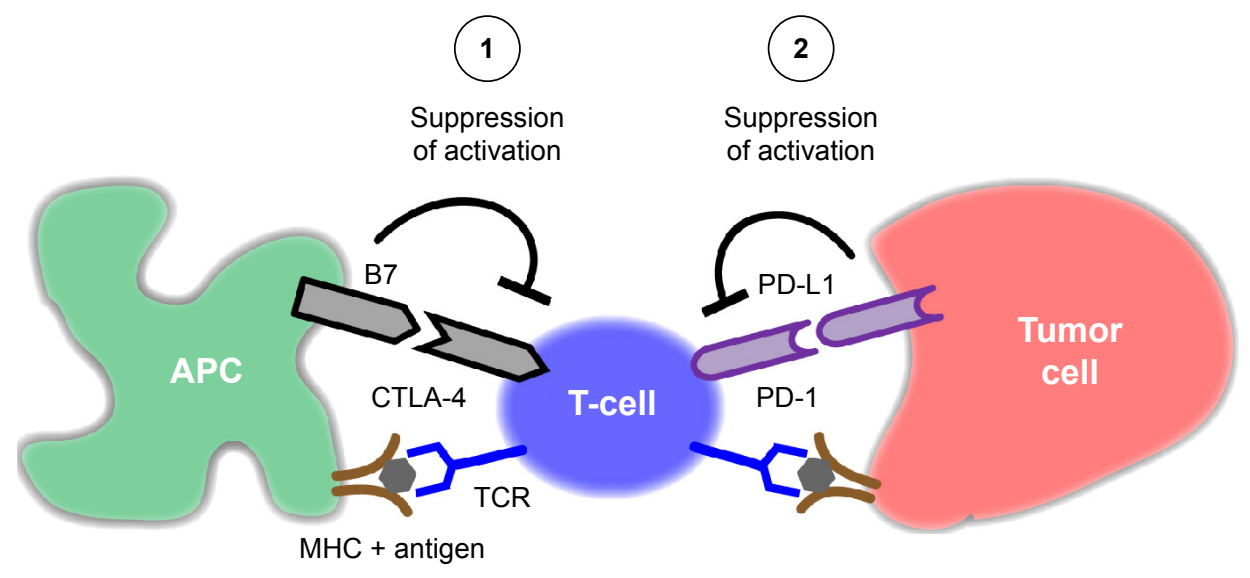

Figure I T-cell activation begins with antigen binding to the TCR complex in conjunction with other costimulatory signals.

Notes: This process can be downregulated at different points. CTLA-4 binding to B7 will block the initial priming of the T-cell (signal I) leading to a suppression of T-cell activation. PD-I regulates immunity at several downstream stages of the immune response. PD-I binding by tumor-elaborated PD-LI (or PD-L2) in peripheral tissues also leads to a suppression of T-cell activation (signal 2). The suppression of T-cell activation is a key step in allowing tumor to evade natural host tumor surveillance and defense.

Abbreviations: TCR, T-cell receptor; CTLA-4, cytotoxic T-lymphocyte-associated antigen; PD-I, programmed cell death-I; PD-LI, programmed death-ligand I; APC, antigen presenting cell; MHC, major histocompatibility complex. 
Table I Targeted therapies in development for modulation of PD-I and PD-LI functions in tumor immune surveillance

\begin{tabular}{ll}
\hline Antibody & Target \\
\hline Pembrolizuma** & PD-I \\
Nivolumab* & PD-I \\
Pidilizumab & PD-I \\
CT-0II & PD-I \\
AMP-224 & PD-I \\
MDX-II05 & PD-LI \\
MPDL3280A & PD-LI \\
MEDI4736" & PD-LI \\
MSB00107I8C & PD-LI \\
\hline
\end{tabular}

Notes: *FDA approved for use in advanced melanomas. "Clinical development indications include advanced melanomas.

Abbreviations: PD-I, programmed cell death I; PD-LI, programmed deathligand I; FDA, US Food and Drug Administration.

Phase I study comprised 173 patients, median age 61 years, with unresectable or metastatic melanoma (referred to herein as advanced melanomas) with disease progression within 24 weeks of ipilimumab treatment and with BRAF inhibitor treatment if $B R A F$-mutant disease. ${ }^{11}$ Patients were randomized into groups that received intravenous pembrolizumab at $2 \mathrm{mg} / \mathrm{kg}(\mathrm{n}=89)$ or $10 \mathrm{mg} / \mathrm{kg}(\mathrm{n}=84)$ every 3 weeks with disease progression or severe toxicity as end points for stopping the treatment. Inclusion criteria dictated that patients did not have known autoimmune disease, any medical condition that required immunosuppressive therapy, or prior severe immune-mediated adverse reactions to ipilimumab treatment. Patients with $B R A F$-mutant disease represented $17 \%$ of the study population. Most (73\%) had received two or more prior therapies for advanced melanoma.

Results were similar across both doses assessed in the study, irrespective of $B R A F$-mutation status. In the lower dose that was ultimately approved, $2 \mathrm{mg} / \mathrm{kg}$, the objective response rate was $24 \%$ ( $95 \%$ confidence interval $=15 \%-34 \%)$. A total of 21 patients showed a response, including one patient with a complete response. Three of the patients (14\%) had disease progression at 2.8 months, 2.9 months, and 8.2 months after the initial response. The other 18 patients $(86 \%)$ showed durable responses of 1.4-8.5 months. Of note, eight patients (38\%) had ongoing responses of $\geq 6$ months.

\section{Efficacy and safety}

A total of $79 \%$ of patients reported drug-related adverse events of any type with $13 \%$ reporting grade 3 or 4 drug-related adverse events. ${ }^{11}$ The most common clinical adverse events of any grade in patients receiving pembrolizumab at $2 \mathrm{mg} /$ $\mathrm{kg}$ were fatigue (47\%), cough (30\%), nausea (30\%), pruritus $(30 \%)$, rash (29\%), and decreased appetite (26\%). Grade 3 or 4 adverse events included fatigue, hypothyroidism, abdominal pain, diarrhea, appetite changes, renal failure, transaminitis, anemia, dyspnea, rash, and pruritus. The most frequent serious adverse events occurring in $\geq 2 \%$ of patients were renal failure, dyspnea, pneumonia, and cellulitis. Immune-related adverse events documented included pneumonitis (4\%), grade 3 or 4 transaminitis (1\%), grade 3 nephritis (1\%), grade 3 hyperthyroidism (1\%), grade 2 adrenal insufficiency (1\%), and hypothyroidism (1\%). Treatment was ultimately discontinued due to adverse events in a total of $6 \%$ of patients.

In approving pembrolizumab, the recommendations included suspending treatment for severe or grade $3+$ treatment-related adverse reactions, with resumption of treatment when adverse reactions recover to grade 0 or 1. Permanent discontinuation is recommended for any life-threatening adverse reactions, including grade 3 or 4 pneumonitis or nephritis and elevated liver enzymes (more than five-fold elevation in alanine transaminase [ALT] and aspartate transaminase [AST]). Persistent grade 2 or 3 events that do not recover to grade 0 or 1 within 12 weeks also necessitate permanent discontinuation. Routine monitoring of thyroid, hepatic, and renal functions is essential in all patients. In April 2015, Phase III randomized control study results were published. ${ }^{15}$ The results showed that pembrolizumab prolonged progression-free survival and overall survival with reduced rates of high-grade toxicity relative to ipilimumab in patients with advanced melanomas.

\section{Future directions}

Cancer therapeutics is undergoing a paradigm shift. The classical approach of targeting the tumor directly has shifted to one in which the patient's immune system is enlisted to unleash its innate tumor eradicating potential. The goal of immunotherapy is restoration of immune function to subvert unbridled tumor growth. The CTLA-4 and PD-1 pathways have garnered unprecedented scrutiny in this regard.

Consistent with the nonredundant function of these two pathways that modulate T-cell activation, it is not surprising that the clinical response to targeting one does not preclude response to targeting the other. Indeed, the observation that patients responded when treated with pembrolizumab after prior failed treatment with ipilimumab establishes that an absence of response to CTLA-4 blockade does not preclude a clinical benefit of PD-1 blockade. ${ }^{11}$ Together, these results suggest that ipilimumab and pembrolizumab might be administered synchronously with a manageable safety profile and perhaps improved efficacy. Confirmation of a more robust and durable response in patients treated with combination therapy requires future studies, but the outlook is encouraging. 


\section{Conclusion}

Immune checkpoint inhibition is at the forefront of antineoplastic drug discovery efforts. Therapies targeting CTLA-4 and PD-1 represent prototypic immune checkpoint inhibitors and have paved the way for more effective PD-1- and PD-L1/ PD-L2-targeted therapeutics in the near future. Ongoing studies are validating that these agents have unequivocal benefit in many solid as well as hematologic malignancies. ${ }^{14}$

In melanoma, the last decade has seen breakthroughs in targeted therapeutics. Inhibitors of MAP kinase signaling, and now PD-1 pathway blockade, bring about the potential for improved patient outcomes. Further research into combination therapies with agents that concomitantly target the MAP kinase pathway while boosting immune surveillance offers an exciting outlook. The FDA approval of pembrolizumab in advanced melanomas carries forth this momentum.

\section{Disclosure}

The authors report no conflicts of interest in this work.

\section{References}

1. Aris M, Barrio MM. Combining immunotherapy with oncogenetargeted therapy: a new road for melanoma treatment. Front Immunol. 2015;6:46.

2. Bhatia S, Tykodi SS, Thompson JA. Treatment of metastatic melanoma: an overview. Oncology (Williston Park). 2009;23(6):488-496.

3. Kee D, McArthur G. Targeted therapies for cutaneous melanoma. Hematol Oncol Clin North Am. 2014;28(3):491-505.
4. Robert C, Karaszewska B, Schachter J, et al. Improved overall survival in melanoma with combined dabrafenib and trametinib. $N$ Engl J Med. 2015;372(1):30-39.

5. Menzies AM, Long GV. Systemic treatment for BRAF-mutant melanoma: where do we go next? Lancet Oncol. 2014;15(9):e371-e381.

6. Rajakulendran T, Adam DN. Bench to bedside: mechanistic principles of targeting the RAF kinase in melanoma. Int J Dermatol. 2014; 53(12):1428-1433.

7. Fedorenko IV, Gibney GT, Sondak VK, Smalley KS. Beyond BRAF: where next for melanoma therapy? Br J Cancer. 2015;112(2): $217-226$.

8. Vinay DS, Ryan EP, Pawelec G, et al. Immune evasion in cancer: mechanistic basis and therapeutic strategies. Semin Cancer Biol. Epub 2015 Mar 25.

9. Shin DS, Ribas A. The evolution of checkpoint blockade as a cancer therapy: what's here, what's next? Curr Opin Immunol. 2015;33C: 23-35.

10. Callahan MK, Wolchok JD. At the bedside: CTLA-4- and PD-1blocking antibodies in cancer immunotherapy. J Leukoc Biol. 2013; 94(1):41-53.

11. Robert C, Ribas A, Wolchok JD, et al. Anti-programmed-deathreceptor-1 treatment with pembrolizumab in ipilimumab-refractory advanced melanoma: a randomised dose-comparison cohort of a phase 1 trial. Lancet. 2014;384(9948):1109-1117.

12. Tumeh PC, Harview CL, Yearley JH, et al. PD-1 blockade induces responses by inhibiting adaptive immune resistance. Nature. 2014; 515(7528):568-571.

13. Postow MA, Callahan MK, Wolchok JD. Immune checkpoint blockade in cancer therapy. J Clin Oncol. 2015;1-9.

14. Momtaz P, Postow MA. Immunologic checkpoints in cancer therapy: focus on the programmed death-1 (PD-1) receptor pathway. Pharmgenomics Pers Med. 2014;7:357-365.

15. Robert C, Schachter J, Long GV, et al. Pembrolizumab versus ipilimumab in advanced melanoma. N Engl J Med. Epub 2015 Apr 19.
Drug Design, Development and Therapy

\section{Publish your work in this journal}

Drug Design, Development and Therapy is an international, peerreviewed open-access journal that spans the spectrum of drug design and development through to clinical applications. Clinical outcomes, patient safety, and programs for the development and effective, safe, and sustained use of medicines are a feature of the journal, which

\section{Dovepress}

has also been accepted for indexing on PubMed Central. The manuscript management system is completely online and includes a very quick and fair peer-review system, which is all easy to use. Visit http://www.dovepress.com/testimonials.php to read real quotes from published authors. 